\title{
The role of comparison in perceptual learning: Effects of concurrent exposure to similar stimuli on the perceptual effectiveness of their unique features
}

\author{
Gabriel Rodríguez, C. A. J. Blair, ANd Geoffrey Hall \\ University of York, York, England
}

\begin{abstract}
In three experiments, rats were given concurrent exposure to a compound flavor (AX) and to one of the elements of the compound $(\mathrm{X})$. The perceptual effectiveness of A was then assessed by a test involving generalization of a conditioned aversion. Comparison was made with a preexposure procedure in which the compound and the common element were presented on separate trials, either in alternation or in separate blocks of trials. The effectiveness of the unique cue was less after blocked preexposure than after either of the other procedures; concurrent preexposure did not produce a greater effect than did alternating preexposure. These results challenge the suggestion that concurrent preexposure engages a special comparison process that will facilitate this form of perceptual learning.
\end{abstract}

According to Gibson (1969), exposure to two or more similar stimuli brings into play a process of differentiation that enhances the perceptual effectiveness (what Hall, 2003, referred to as the effective salience) of the unique stimulus features and reduces that of the common features. This sort of change in salience will enhance the perceptual dissimilarity of the stimuli, thus enhancing their discriminability (i.e., reducing generalization between them and generating a perceptual learning effect). Importantly, Gibson (e.g., 1969, p. 145) emphasized the suggestion that differentiation will be particularly likely to operate under conditions promoting stimulus comparison. Over recent years, these proposals have been investigated in experiments using the flavor-aversion learning procedure. For instance, Symonds and Hall (1995, Experiment 2) gave rats preexposure to two flavor compounds - AX and BX (where A and B represent unique features of the stimuli and $\mathrm{X}$ represents an explicitly added common feature) - in alternating trials (AX, BX, AX, BX ...). Control subjects received an equivalent amount of preexposure to the stimuli, but in a schedule in which AX and BX were presented in separate blocks of trials (e.g., AX, AX ..., BX, BX ...). For all subjects, an aversion was then established to AX and generalization to $\mathrm{BX}$ was tested. It was found that rats given alternating preexposure showed less generalization (i.e., a better discrimination) between $\mathrm{AX}$ and $\mathrm{BX}$ than did those that received blocked preexposure (see also, e.g., Bennett \& Mackintosh, 1999; Honey, Bateson, \& Horn, 1994; Mondragón \& Hall, 2002).
More recently, results from other procedures have lent support to the proposal that alternating preexposure will enhance the effective salience of the unique features of the stimuli. It has been shown that a unique feature presented according to the alternating schedule is more effective than one presented according to the blocked schedule in eliciting its unconditioned response (UR; Blair, Wilkinson, \& Hall, 2004, Experiment 1), as an unconditioned stimulus (US) in classical conditioning (Blair et al., 2004, Experiment 2), and as a conditioned stimulus (CS) in classical conditioning (Blair et al., 2004, Experiment 3; Mondragón \& Hall, 2002, Experiment 4; see also Lombas, Alonso, \& Rodríguez, 2008). Such a feature is also particularly effective in interfering with the conditioned response (CR) controlled by some other stimulus (Blair \& Hall, 2003, Experiment 5) when the feature and this other stimulus are presented in compound.

What these experiments do not demonstrate, however, is that the effect produced by the alternating preexposure schedule is a consequence of stimulus comparison. We may agree that a comparison process is likely to operate when two stimuli are presented repeatedly in alternation and in quick succession; however, the schedule used for alternating preexposure in the experiments just described was very different from this. There were rather few stimulus presentations (usually four of each), and the minimum interval between exposure to one flavor and the presentation of the next was several hours. Comparison - as it is generally understood, which would involve experiencing one stimulus in the presence of another (or in the pres- 
ence of the immediate aftereffects of the other) - seems unlikely to operate here. A role for comparison would be better demonstrated if it could be shown that the differentiation process occurs most readily when the stimuli are preexposed in close succession.

This proposal has been investigated in a number of experiments in which AX and BX were presented simultaneously. Subjects under this concurrent schedule would have the opportunity to sample the flavors in quick succession; thus, the conditions for comparison to occur (and for differentiation to operate) should be better than those in the standard alternating (or blocked) schedule. What follows from this suggestion is that two stimuli should be perceived as being more dissimilar - and, thus, generalization between them should be more reduced - after concurrent preexposure than after alternating or blocked exposure. However, tests of this prediction using the flavor-aversion learning procedure have shown that, far from reducing generalization, concurrent preexposure actually results in more generalization than does an alternating (Bennett \& Mackintosh, 1999, Experiment 2; Rodríguez \& Alonso, 2008) or a blocked (Alonso \& Hall, 1999; Rodríguez \& Alonso, 2008) schedule. That is, conditions that appear ideal for comparing the stimuli resulted in subjects showing an apparently reduced capacity to discriminate between them.

Although the most obvious interpretation of these results is that this aspect of Gibson's (1969) analysis of perceptual learning is wrong, there is an alternative interpretationfirst noted by Honey et al. (1994) - that may be able to rescue her account. The possibility is that differentiation occurs readily during concurrent preexposure, but that it fails to manifest itself as a generalization-reducing effect because it is counteracted by the opposite influence of some generalization-enhancing processes. The most obvious of these is sensory preconditioning. The simultaneous presentation of a pair of stimuli (such as AX and BX) could allow the formation of excitatory associations between their unique features (A and B). Consequently, when animals given concurrent preexposure are tested after conditioning to $\mathrm{AX}$, the presentation of $\mathrm{BX}$ could activate (by way of the B-A association) the representation of A, which would be itself associated with the US, thus increasing the aversion observed (i.e., enhancing the generalization between AX and BX). This source of generalization would not be available to animals given alternating or blocked preexposure, for whom the longer interval between the presentations of $\mathrm{AX}$ and $\mathrm{BX}$ would serve to preclude the formation of direct excitatory associations between $\mathrm{A}$ and $\mathrm{B}$.

In order to eliminate the effect of sensory preconditioning, Rodríguez and Alonso (2008) investigated the effects of concurrent preexposure not to $\mathrm{AX}$ and $\mathrm{BX}$, but to $A X$ and $X$. If the more profound generalization found after concurrent preexposure to AX and BX (Alonso \& Hall, 1999; Bennett \& Mackintosh, 1999) depends on the formation of an excitatory association between A and B, then no such effect should be expected in a procedure in which the B feature is omitted; however, a comparison process should still be able to enhance the effectiveness of the unique A feature and thus reduce generalization between the preexposed cues. Rodríguez and Alonso (2008) gave three groups of rats exposure to $\mathrm{AX}$ and $\mathrm{X}$ in these arrangements: concurrently, in alternation, and on separate blocks of trials. All animals then received aversion conditioning trials, with $\mathrm{X}$ as the CS. Subsequent testing revealed that generalization of this aversion to AX was less in animals that were given alternating exposure than in those given blocked exposure (thus confirming that the presence of a unique feature - e.g., B - in one of the tobe-discriminated stimuli is not essential to produce the alternating versus blocked effect; see also Hall, Blair, \& Artigas, 2006; Rodríguez \& Alonso, 2004). But the test also showed that even with sensory preconditioning neutralized, conditioned responding to AX was still more profound in animals given concurrent exposure than in those given either alternating or blocked exposure.

Importantly, however, this effect was accompanied by a stronger conditioned aversion to $\mathrm{X}$ in the concurrent than in the other preexposure conditions (see also Bennett \& Mackintosh, 1999, Experiment 2), perhaps because simultaneous presentations of the stimuli (AX and X) limited the development of latent inhibition to the common feature (X). This in itself would be enough to explain the greater responding to AX observed after concurrent preexposure. It follows that in order to provide a fair test of the effects of these different forms of preexposure on generalization, it is necessary for one to both neutralize the influence of sensory preconditioning in the concurrent condition and also ensure that the associative strength of the CSagainst which generalization is assessed - is equivalent in all conditions. This is what we attempted to accomplish in the present experiments.

\section{EXPERIMENT 1}

In Experiment 1 (for a summary of the experimental designs, see Table 1), all rats received preexposure trials with two compound flavor stimuli- $\mathrm{AX}$ and $\mathrm{BX}$ - and with the common element of these compounds, X. Two drinking tubes were made available to the rats on each exposure session. In one of the two daily sessions, one tube contained AX and the other contained X. In the other daily

\begin{tabular}{|c|c|c|}
\hline \multicolumn{3}{|c|}{$\begin{array}{c}\text { Table 1 } \\
\text { Experimental Designs }\end{array}$} \\
\hline Preexposure & Conditioning & Test \\
\hline \multicolumn{3}{|c|}{ Experiment 1} \\
\hline $\mathrm{AX}-\mathrm{X} / \mathrm{BX}-\mathrm{W}$ & $\mathrm{X}+$ & $\mathrm{AX}$ and $\mathrm{BX}$ \\
\hline \multicolumn{3}{|c|}{ Experiment 2} \\
\hline $\mathrm{AX}-\mathrm{X} / \mathrm{BX}-\mathrm{W}$ & $\mathrm{Y}+$ & $\mathrm{AY}$ and $\mathrm{BY}$ \\
\hline \multicolumn{3}{|c|}{ Experiment 3} \\
\hline \multicolumn{3}{|l|}{$\mathrm{CNC}$ group } \\
\hline $\mathrm{AX}-\mathrm{X} / \mathrm{AX}-\mathrm{X}$ & $\mathrm{Y}+$ & $\mathrm{AY}$ \\
\hline \multicolumn{3}{|l|}{ ALT group } \\
\hline $\mathrm{AX}-\mathrm{AX} / \mathrm{X}-\mathrm{X}$ & $\mathrm{Y}+$ & $\mathrm{AY}$ \\
\hline \multicolumn{3}{|l|}{ BLK group } \\
\hline $\mathrm{AX}-\mathrm{AX} / \mathrm{AX}-\mathrm{AX} \ldots \mathrm{X}$ & $\mathrm{Y}+$ & AY \\
\hline
\end{tabular}

Note- $\mathrm{A}, \mathrm{B}, \mathrm{X}$, and $\mathrm{Y}$ refer to flavors; $\mathrm{W}$ refers to water. During preexposure, stimuli separated by a dash (-) were presented simultaneously within the same trial, and those separated by a forward slash (/) were presented on alternate trials within a day; + refers to the administration of LiCl. 
session, one tube contained $\mathrm{BX}$ and the other contained water. That is, presentations of $\mathrm{AX}$ and $\mathrm{X}$ were given according to a concurrent schedule (both being presented simultaneously), and presentations of BX and X (and those of $\mathrm{AX}$ and $\mathrm{BX}$ ) were given according to an alternating schedule (one stimulus being presented in the morning and the other in the afternoon, or vice versa). For all subjects, discrimination between $\mathrm{X}$ and $\mathrm{AX}$ and between $\mathrm{X}$ and $\mathrm{BX}$ was then assessed by establishing an aversion to $\mathrm{X}$ and measuring generalization of this aversion to $\mathrm{AX}$ and $\mathrm{BX}$. If the opportunity for comparison facilitates differentiation and generates perceptual learning, then it might be expected that the generalized aversion from $\mathrm{X}$ to AX (preexposed concurrently) would be less profound than that from X to BX (preexposed in alternation).

This experimental design, we thought, would allow a test of the effects of concurrent preexposure that was free from the effects of the complicating factors described above. First, omitting the unique feature of one of the two stimuli to be discriminated (Stimulus X) should — on the face of things - preclude any contribution from sensory preconditioning. And second, the use of a within-subjects design should neutralize the role of latent inhibition to the common features on generalization. Whatever the contribution made by the aversion acquired by $\mathrm{X}$ during conditioning to the performance shown on test, it must be assumed to be the same on both the $\mathrm{AX}$ and the BX tests.

\section{Method}

Subjects and Apparatus. The subjects were 16 male hooded Lister rats with a mean ad lib weight of $414 \mathrm{~g}$ at the start of the experiment. The animals had previously been used in another experiment; however, they were naive to all aspects of the present procedure. The rats were singly housed with continuous access to food in a colony room that was artificially lit from 8:00 a.m. to 8:00 p.m. each day. Access to water was restricted as detailed below.

The solutions used as experimental stimuli were administered in the home cages at room temperature in 50-ml plastic centrifuge tubes, each equipped with a rubber stopper to which was fitted a stainless steel, ball-bearing-tipped spout. The following flavored solutions were used: a $0.16 \mathrm{M}$ saline $(\mathrm{NaCl})$ solution, a compound consisting of $0.16 \mathrm{M}$ saline and vanilla $(1 \% \mathrm{w} / \mathrm{v}$ vanilla flavoring supplied by SuperCook, Leeds, U.K.), and a compound of $0.16 \mathrm{M}$ saline and almond (2\% w/v SuperCook almond flavoring). Consumption was measured by weighing the tubes before and after trials to the nearest $0.1 \mathrm{~g}$. The US for the conditioning trials was an intraperitoneal injection of $0.15 \mathrm{M}$ lithium chloride $(\mathrm{LiCl})$ at $10 \mathrm{ml} /$ $\mathrm{kg}$ of body weight.

Procedure. The water-deprivation regime was initiated by removing the standard water bottles overnight. On each of the following 4 days, access to water was restricted to two daily sessions of $30 \mathrm{~min}$, at 11:00 a.m. and 5:00 p.m. The presentation of fluids continued to be given at these times throughout the experiment.

Over the next 4 days (the preexposure phase), all subjects were given access to fluid in two drinking tubes (separated from one another by $5 \mathrm{~cm}$ ), each containing $5 \mathrm{ml}$ of the appropriate fluid (AX, $\mathrm{BX}, \mathrm{X}$, or water). In one of the two daily drinking sessions, one tube contained $5 \mathrm{ml}$ of $\mathrm{AX}$ and the other $5 \mathrm{ml}$ of $\mathrm{X}$; in the other daily session, one tube contained $5 \mathrm{ml}$ of BX and the other $5 \mathrm{ml}$ of water. The left-right position of the tubes was counterbalanced. For half of the subjects, $\mathrm{AX}$ and $\mathrm{X}$ were presented in the morning session, and $\mathrm{BX}$ was presented in the afternoon session; for the remainder, the arrangement was reversed. Half of the subjects received vanilla as A and almond as B; for the rest, the arrangement was reversed. For all animals, $\mathrm{X}$ was saline.
Two conditioning trials followed. The first was given in the morning session the day after preexposure ended. It consisted of a 30-min presentation of the two drinking tubes, each containing $5 \mathrm{ml}$ of X, followed immediately by an injection of $\mathrm{LiCl}$. The rats were given free access to water in the afternoon session. The next day was a recovery day on which animals had unrestricted access to water for $30 \mathrm{~min}$ during both morning and afternoon sessions. The second conditioning trial — given in the morning session of the next day — was identical to the first and was followed by an additional recovery day.

On the following morning session, subjects were given a freeaccess test trial that consisted of a 30-min presentation of the two drinking tubes. For half of the animals, both tubes contained AX, and for the remainder, they contained BX. Water was made available in the afternoon session. The next morning, animals that had been tested with AX the previous day were given an identical test trial with BX, and vice versa. This schedule of testing was continued for another 6 days, making a total of four presentations of AX and four of BX.

\section{Results and Discussion}

The rats consumed almost all of the fluid made available on each of the preexposure sessions $(5 \mathrm{ml}$ of each solution). Average consumptions of AX, BX, X, and water over the course of preexposure were $4.5,4.4,4.6$, and $4.4 \mathrm{ml}$, respectively. Conditioning trials successfully established an aversion to X. The mean amounts consumed were $9.18 \mathrm{ml}$ on Trial 1, $12.08 \mathrm{ml}$ on Trial 2, and $3.65 \mathrm{ml}$ on Trial 3, with all subjects showing a reduction from the second trial to the third.

Figure 1 shows group mean consumption over the test trials with $\mathrm{AX}$ and $\mathrm{BX}$. Levels of consumption were low on the initial trials, but recovered with continued testing. Consumption of BX was greater than that of AX throughout the test. An ANOVA conducted on the data (summarized in Figure 1) confirmed there to be a significant effect of test stimulus $(\mathrm{AX}$ or $\mathrm{BX})[F(1,15)=6.88$; here and elsewhere, a significance level of $p<.05$ was adopted] and a significant effect of trial $[F(5,75)=35.56]$. The interaction between these two factors was not significant $[F(5,75)=1.29]$.

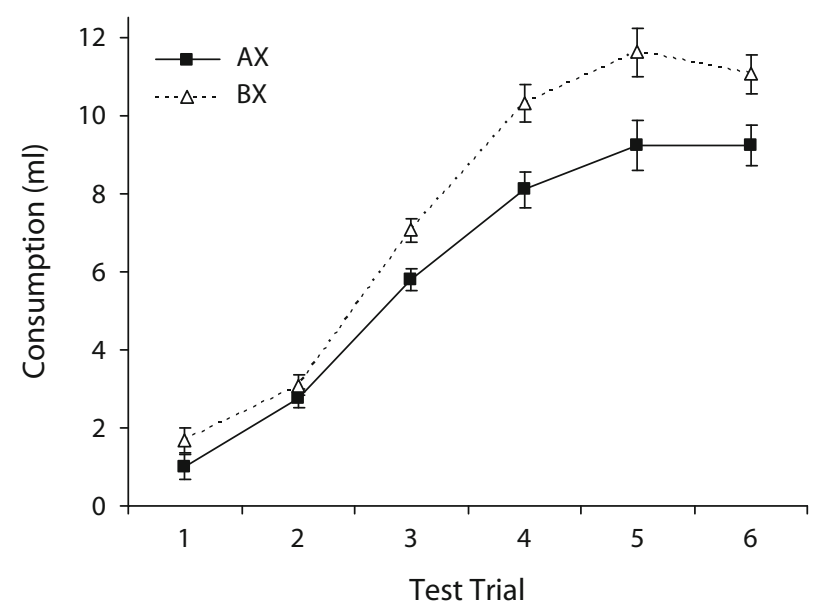

Figure 1. Experiment 1: Mean consumption of the flavor compounds $\mathrm{AX}$ and $\mathrm{BX}$ after aversion conditioning with $\mathrm{X}$. Animals had received preexposure consisting of the simultaneous presentation of $A X$ and $X$ on one of the two daily trials and presentation of BX on the other. Vertical bars represent within-subjects standard errors computed on scores adjusted for variation between subjects (Bakeman \& McArthur, 1996). 
These results show that generalization from the CS (X) occurred more readily to AX (which was presented concurrently with $\mathrm{X}$ during preexposure) than to $\mathrm{BX}$, the presentations of which were given in alternation with those of X. This outcome parallels those obtained previously in studies using a between-subjects comparison between groups given concurrent and alternating preexposure (Bennett \& Mackintosh, 1999; Rodríguez \& Alonso, 2008). It was argued above that the present experimental design would neutralize the two generalization-enhancing processes (sensory preconditioning and the attenuation of latent inhibition to the stimulus common features) potentially responsible for these previous results. Why, then, was generalization still more profound after concurrent preexposure? We had anticipated that a comparison process engaged by concurrent exposure might reduce generalization to AX, or, if no such process operates, that there would be no difference in performance to AX and BX. That the generalization was greater to AX than to BX requires explanation.

One possibility is that we were wrong in assuming that our experimental procedure fully neutralized the contribution of sensory preconditioning on generalization. Perhaps the purely elemental analysis of this phenomenon that we adopted led us to underestimate it. It might be argued that presentations of each of the preexposed stimuli (AX, BX, and $\mathrm{X}$ ) activated a distinct configural representation of them in addition to (or instead of) a representation of each of their component elements. If this is true, then the simultaneous presentations of $\mathrm{AX}$ and $\mathrm{X}$ during preexposure would have allowed the formation of an excitatory association between the configural representation of $\mathrm{AX}$ and that of X. What follows is that, on test, the presentation of AX would be able to retrieve a representation of the X configuration that would have acquired some strength during conditioning with $\mathrm{X}$ as the $\mathrm{CS}$, thus increasing the aversion observed. An implication of this hypothesis is that if $\mathrm{X}$ is not present during conditioning, then this sensory preconditioning effect should not produce any effect on generalization. The next experiment evaluated this implication.

\section{EXPERIMENT 2}

In Experiment 2 (its design is outlined in Table 1), the preexposure treatment was identical to that of Experiment 1, but critically (in order to neutralize the contribution of the sensory preconditioning), a novel stimulus (Y) was used as the CS during the conditioning phase and was then presented in compound with A or B on test. Excitatory associations might be formed during preexposure between the (configural) representations of $\mathrm{AX}$ and $\mathrm{X}$, but these would not be relevant to a test given with the AY compound.

With sensory preconditioning neutralized, the present design should be effective in revealing any difference in salience between the unique stimulus features $\mathrm{A}$ and $\mathrm{B}$. If the opportunity for comparison renders A more salient than B, then Stimulus A should be more effective than $\mathrm{B}$ in interfering with the expression of the aversive CR established to Y, and consumption of AY on test should be greater than consumption of BY.

\section{Method}

The subjects were 16 male hooded Lister rats with a mean ad lib weight of $430 \mathrm{~g}$ at the start of the experiment. The rats had previously been used in another experiment; however, they were naive to all aspects of the present procedure. This experiment was identical to Experiment 1, except that conditioning was given with a solution of $0.00003 \mathrm{M}$ quinine sulfate $(\mathrm{Y})$ as the $\mathrm{CS}$, and test was given with a compound consisting of $0.00003 \mathrm{M}$ quinine sulfate and vanilla $1 \%$ $\mathrm{w} / \mathrm{v}$, and a compound consisting of $0.00003 \mathrm{M}$ quinine sulfate and almond $2 \% \mathrm{w} / \mathrm{v}$ (AY and BY, counterbalanced). In details not specified here, the procedure was the same as that described for Experiment 1.

\section{Results and Discussion}

As in Experiment 1, the rats drank almost all of the fluid made available on each of the preexposure sessions. The mean scores for consumption of AX, BX, X, and water were 4.6, 4.6, 4.4, and $4.4 \mathrm{ml}$, respectively. The conditioning trials successfully established an aversion to Y. The mean amounts consumed were $8.95 \mathrm{ml}$ on Trial $1,1.34 \mathrm{ml}$ on Trial 2, and $0.64 \mathrm{ml}$ on Trial 3, with all subjects showing a reduction from one trial to the next. Figure 2 shows group mean consumption over the test trials with AY and BY. As was the case in Experiment 1, consumption was suppressed on the initial test trials, but in this case, the levels of consumption of both AY and BY progressively increased at a similar rate. An ANOVA conducted on the data summarized on Figure 2 yielded a significant main effect of trial $[F(5,75)=81.18]$. Neither the main effect of test stimulus (AY or BY) nor the trial $\times$ test stimulus interaction was significant $\left(F_{\mathrm{S}}<1\right)$.

There is thus no support for the hypothesis that the different preexposure treatments given to $\mathrm{A}$ and $\mathrm{B}$ will render them different in their effective salience. The absence of any difference in levels of consumption of the test stimuli in this experiment prompts the conclusion that the effect seen in Experiment 1 was probably a consequence of some form of sensory preconditioning requiring conditioning

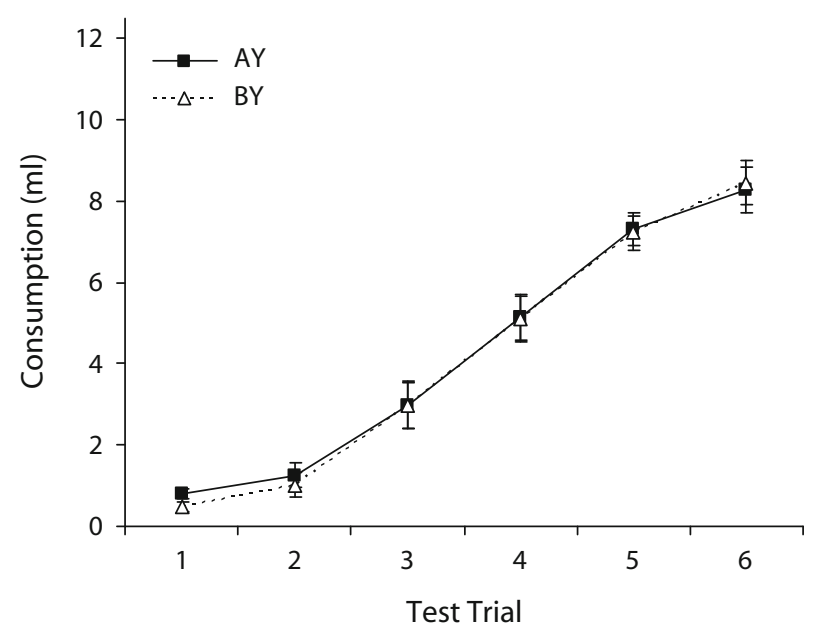

Figure 2. Experiment 2: Mean consumption of the flavor compounds $A Y$ and $B Y$ after aversion conditioning with $Y$. Animals had received preexposure consisting of the simultaneous presentation of $A X$ and $X$ on one of the two daily trials and presentation of BX on the other. Vertical bars represent within-subjects standard errors. 
to Stimulus $\mathrm{X}$, which was neutralized in the present experiment by giving conditioning to $\mathrm{Y}$ and testing with $\mathrm{AY}$ and BY. Furthermore, the lack of difference between the consumption of AY and BY indicates (as well as any null result can) that A and B were equally effective (or ineffective) in interfering with the aversion controlled by Y. However, since it is known from other experiments (see below) that the alternating schedule is effective in this respect, this null result points to a more positive conclusion - that the concurrent schedule is equally, but not more, effective in modulating the effective salience of the unique stimulus features. There is no need, therefore, to postulate that concurrent preexposure engages a comparison process different from any that might operate during simple alternation schedule. Confirmation of this proposal required an additional experiment with an added control condition.

\section{EXPERIMENT 3}

Evidence supporting the notion that alternating preexposure enhances the effective salience of the unique stimulus features comes from studies comparing the effects of alternating and blocked preexposure schedules (e.g., Blair \& Hall, 2003; Blair et al., 2004; Mondragón \& Hall, 2002). If - as our interpretation of results of Experiment 2 suggests - concurrent and alternating preexposure are equally effective in enhancing effective salience, then the effect should be evident in both when comparison is made with subjects exposed to an equivalent blocked preexposure regime. To address this, we conducted Experiment 3, which maintained the essential features of Experiment 2 (i.e., conditioning with a novel cue Y prior to the test) but made use of a between-subjects design.

As in the previous experiments, two drinking tubes were made available to the rat on each experimental session. There were three groups that differed in the preexposure they received. Group Concurrent $(\mathrm{CNC})$ received preexposure in which one tube contained $\mathrm{AX}$ and the other X. Groups Alternating (ALT) and Blocked (BLK) received equivalent preexposure to the flavors; however, for them, both tubes contained the same flavor (either AX or X) on each trial. Group ALT received AX and X on alternate trials; Group BLK received AX in the first block of trials and $\mathrm{X}$ in the second, or vice versa. All rats then received aversion conditioning with $\mathrm{Y}$ as the $\mathrm{CS}$, followed by generalization tests with AY. As before, since $\mathrm{X}$ was not presented in either the conditioning or the test phase, the contribution of sensory preconditioning in the CNC group should be eliminated, and any differences between the groups in degree of latent inhibition to $\mathrm{X}$ would be irrelevant. If concurrent and alternating preexposure schedules are equally effective in enhancing the salience of the unique feature A, then Groups CNC and ALT should drink AY equally readily on test, and both should show greater consumption of AY than would Group BLK.

\section{Method}

The subjects were 24 male hooded Lister rats with a mean ad lib weight of $483 \mathrm{~g}$ at the start of the experiment. The rats had previously been used in another experiment; however, they were naive to all aspects of the present procedure. They were maintained in the same way and on the same water deprivation schedule as was described for Experiment 1.

The rats were divided into three equal-sized groups. Over the next 4 days (the preexposure phase), all were given two daily sessions in which they received fluids in two drinking tubes, each containing $5 \mathrm{ml}$ of the appropriate fluid (AX or X). For Group CNC, one tube contained $\mathrm{AX}$, and the other contained $\mathrm{X}$, on each session. For Groups ALT and BLK, the two drinking tubes contained the same solution on each session. Animals in Group ALT were given access to the flavors in alternation. For half of the animals in this group, AX was presented during the morning session and $\mathrm{X}$ in the afternoon; for the remainder, the arrangement was reversed. Animals in Group BLK received the solutions in two blocks of trials. For half of the animals in this group, AX was presented on the first 2 days in both daily sessions and $X$ on the last 2 days. The remaining animals received the opposite sequence.

After preexposure, all three groups received two conditioning trials with $\mathrm{Y}$ as the CS, according to the procedure described for Experiment 2. Following the final recovery day, the animals received five test trials with AY over 5 consecutive days. In details not specified here, the procedure was the same as that described for the previous experiments.

\section{Results and Discussion}

The rats consumed all of the fluid offered throughout the preexposure phase. The conditioning trials successfully established an aversion to Y. On the first conditioning trial, all rats drank less than the $10 \mathrm{ml}$ made available (the mean amounts consumed were 7.5, 7.8, and $7.2 \mathrm{ml}$, for Groups CNC, ALT, and BLK, respectively). Consumption was suppressed on the second trial (the means were $3.3 \mathrm{ml}$ for Group CNC, $3.8 \mathrm{ml}$ for Group ALT, and $2.7 \mathrm{ml}$ for Group BLK). An ANOVA conducted with these datawith group and trial as the variables - showed there to be a significant effect of trial $[F(1,21)=148.59]$. Neither the main effect of group $[F(2,21)=1.74]$ nor the trial $\times$ group interaction was significant $[F(2,21)<1]$.

Figure 3 shows the group mean consumption over the generalization tests with AY. The level of consumption was low on the initial trials, although consumption of $\mathrm{AY}$ was marginally greater in Groups CNC and ALT than in Group BLK. This difference among the groups increased with continued testing, and statistical analysis confirmed this impression. An ANOVA conducted on the data summarized in Figure 3 showed there to be a significant effect of trial $[F(4,84)=66.84]$, no significant effect of group $[F(2,21)=3.11]$, but a significant interaction between trial and group $[F(8,84)=2.22]$. Pairwise comparisons using the $t$ test confirmed that Group CNC drank significantly more than did Group BLK on Trial 3 and that Groups CNC and ALT drank significantly more than did Group BLK on Trials 4 and 5. This is just the pattern of results predicted by the hypothesis that motivated Experiment 3. It is consistent with the view that $\mathrm{A}$ interfered to a greater extent with the aversion controlled by $\mathrm{Y}$ in Groups CNC and ALT than in Group BLK. We conclude that, using a procedure in which sensory preconditioning and latent inhibition to the stimulus common features are fully neutralized, concurrent and alternating preexposure schedules do not differ, but both are more effective than the blocked schedule in enhancing the salience of the stimulus unique features. The implications of this finding will be considered next. 


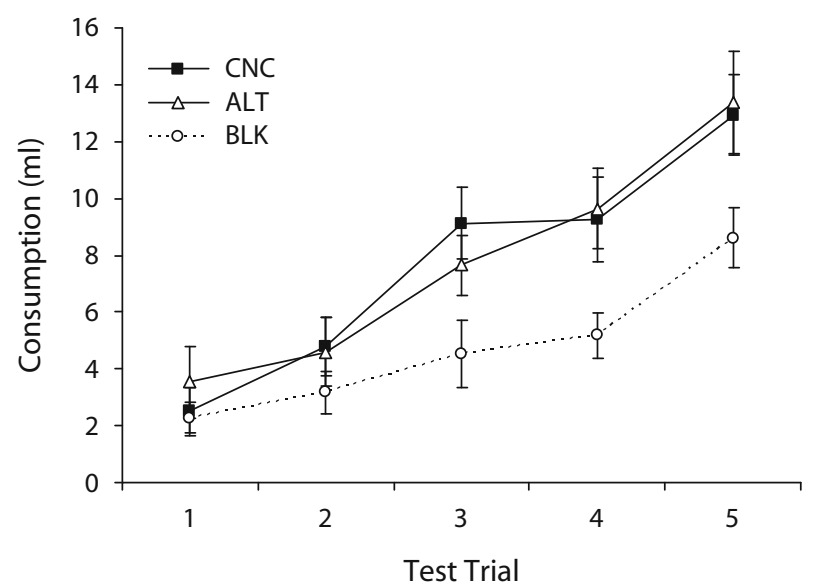

Figure 3. Experiment 3: Group mean consumption of the compound flavor AY after aversion conditioning with Y. Group CNC had received concurrent preexposure to $\mathbf{A X}$ and $X$. Group ALT had received preexposure to $A X$ and $X$ in alternating trials. Group BLK had received preexposure to $A X$ and $X$ on separate blocks of trials. Vertical bars represent the standard errors of the means.

\section{GENERAL DISCUSSION}

It is a common (sadly, perhaps not everyday) experience that discrimination between two wines can be enhanced by taking alternate sips of each in fairly close succession. In these circumstances, the distinctive features of each become apparent in a way that would not otherwise be possible. It is this sort of experience- as much as any prediction from formal theorizing - that leads to the proposal that perceptual learning effects will be more evident when the stimuli to be discriminated are presented concurrently in the preexposure phase. This proposal was tested in experiments in which rats were given concurrent exposure to two similar fluids, one of which had an added distinctive feature. They were then given a test that required discrimination between a subsequently trained CS and a compound of this CS and the distinctive feature. Enhancement of the perceptual effectiveness of the feature might be expected to improve discrimination (i.e., reduce generalization between the CS and the compound).

The results of these experiments lend no support to the proposal. Preexposure in which the stimuli were presented in alternation was found to be more effective in limiting generalization than preexposure in which they were presented in separate blocks of trials (confirming a result well established by previous research). However, in the alternating procedure, presentations of the stimuli were spaced several hours apart, and reducing this interval by making them available concurrently did not enhance discrimination. Indeed, in Experiment 1, concurrent exposure was found to enhance generalization between the two flavors - a result that we attributed to the formation of excitatory associations between the cues during preexposure (see also Alonso \& Hall, 1999). When this effect was controlled for (Experiments 2 and 3), the difference between the concurrent and alternating preexposure schedules was eliminated, although both showed an advantage in comparison with the blocked schedule (Experiment 3 ).

Current explanations for the differing effects of the alternating and blocked schedules have been based on associative principles (Hall, 2003; McLaren \& Mackintosh, 2000) - specifically, in terms of effects produced by the formation of associations among the various components of the compound stimuli used in these procedures. In the version proposed by Hall (2003), the critical factor is that the presentation of $\mathrm{X}$ allows for the associative activation of A by way of the X-A association formed on an earlier trial. It is this associative activation that is proposed to maintain the effective salience of A. From this perspective, the absence of a difference between the concurrent and alternating schedules is to be expected. Given the standard assumption that associative links tend to be long lasting, there is no reason why the rapid alternation allowed by the concurrent schedule should be more effective than alternation in which the stimulus presentations are well spaced. To this extent, the present results are consistent with current theorizing. It could be argued, however, that an account that emphasizes the number of alternations between AX and $\mathrm{X}$ - rather than the interval between them - might still expect a difference between the CNC and ALT groups of Experiment 3. Concurrent preexposure will result in more alternations if the rat switches frequently between the tubes. This will still be true even if - as our informal observations indicate - the rats in the concurrent condition tend to drink all of one solution before switching to the other. Under these conditions, there are likely, on average, to be twice as many alternations in the concurrent condition as there are in the alternating condition. The absence of a difference must mean that one or another of our assumptions is wrong. The most obvious is that this analysis has failed to take account of the fact that on any given X trial, the animals in the ALT group will have drunk twice the amount of AX on the preceding trial than what was available to animals in the $\mathrm{CNC}$ group. The stronger $\mathrm{X}-\mathrm{A}$ association generated by this more extensive exposure to AX could well compensate for the reduced number of alternations experienced in the ALT condition.

It remains the case, however, that facilitated discrimination as a result of concurrent exposure appears to be-as we have already noted - a fact of common experience, and there is some experimental evidence from early studies of animal discrimination learning that appears to substantiate this claim. Spence's (1936) account of discrimination learning, which dealt only in terms of the acquisition of associative strength by individual cues, was challenged by attempts to show that simultaneous discrimination procedures were more effective than successive training procedures, the suggestion being that the former allowed the possibility of comparison between the cues, whereas the latter did not. The proper interpretation of many of these studies is debatable (see Sutherland \& Mackintosh, 1971, for a review); however, one study by Saldanha and Bitterman (1951) seems to make the point. In this study, rats were trained on two simultaneous discriminations concurrently. One group received trials on which choice lay between two different shades of gray, intermixed with trials on which 
the choice was between wide and narrow stripes. A second group received trials in which the positive gray was paired with the nonrewarded stripe width, intermixed with other trials in which the positive stripe width was presented with the nonrewarded gray. The first group thus got the chance to compare each pair of similar stimuli, whereas the second group did not. Saldanha and Bitterman found that the first group learned the discrimination more readily than did the second group, and they concluded that comparison promoted discrimination learning.

It should be noted, however, that this result does not necessarily imply that comparison facilitates perceptual learning. It suggests that the opportunity for comparison available to the first group made them better able to perceive the distinctive features of the cues (and thus better able to form the appropriate associations with reward and nonreward), but it does not demonstrate the operation of a learning process that produces some longer term change in the perceptual effectiveness of the stimuli. In a more recent study with pigeons as the subjects, Wills and Mackintosh (1999) made use of the same basic design, the stimulus pairs being rectangles that differed in luminance and stars that differed in the number of points they possessed. In their Experiment 1, they found that the opportunity for comparison bestowed an advantage, but only in the case of the luminance discrimination. They concluded that this effect was best explained in terms of the operation of a low-level sensory process that - at least for some stimulus dimensions (and luminance is an obvious candidate) - allows the contrast between simultaneously presented, similar stimuli to enhance the perceived difference between them. This enhancement would facilitate the acquisition of the associations required for discriminative performance, but it would not necessarily produce longer term changes in the effectiveness of the stimuli. In another experiment, Wills and Mackintosh showed that the beneficial effect of initial training on a simultaneous luminance discrimination was not sustained when the pigeons were given a test in which the stimuli were presented individually rather than concurrently.

These considerations help to resolve the apparent discrepancy between the present results and the intuitions that arise from everyday experience. Comparison between two flavors presented repeatedly in quick succession may indeed facilitate immediate discrimination between them during the course of their presentation. However, the effects need not be evident when the subjects are required to make a discrimination some time later. Perceptual learning effects can be seen on such a test, but these are generated as readily by exposure in which the stimulus presentations are widely spaced as by exposure in which they are presented concurrently.

\section{AUTHOR NOTE}

This research was supported by a grant from the United Kingdom Biotechnology and Biological Sciences Research Council and was conducted while G.R. was supported by a postdoctoral fellowship from the
Basque Government (Programa de Formación de Investigadores del Departamento de Educación, Universidades e Investigación). Correspondence concerning this article should be addressed to G. Rodríguez, Department of Psychology, University of York, York YO10 5DD, England (e-mail: gr509@york.ac.uk).

Note-This article was accepted by the previous editorial team, when Shepard Siegel was Editor.

\section{REFERENCES}

Alonso, G., \& Hall, G. (1999). Stimulus comparison and stimulus association processes in the perceptual learning effect. Behavioural Processes, 48, 11-23.

BAKeman, R., \& McArthur, D. (1996). Picturing repeated measures: Comments on Loftus, Morrison, and others. Behavior Research Methods, Instruments, \& Computers, 28, 584-589.

Bennett, C. H., \& Mackintosh, N. J. (1999). Comparison and contrast as a mechanism of perceptual learning? Quarterly Journal of Experimental Psychology, 52B, 253-272.

Blair, C. A. J., \& Hall, G. (2003). Changes in stimulus salience as a result of stimulus preexposure: Evidence from aversive and appetitive testing procedures. Learning \& Behavior, 31, 185-191.

Blair, C. A. J., Wilkinson, A., \& Hall, G. (2004). Assessments of changes in the effective salience of stimulus elements as a result of stimulus preexposure. Journal of Experimental Psychology: Animal Behavior Processes, 30, 317-324.

Gibson, E. J. (1969). Principles of perceptual learning and development. New York: Appleton-Century-Crofts.

HALL, G. (2003). Learned changes in the sensitivity of stimulus representations: Associative and nonassociative mechanisms. Quarterly Journal of Experimental Psychology, 56B, 43-55.

Hall, G., Blair, C. A. J., \& Artigas, A. A. (2006). Associative activation of stimulus representations restores lost salience: Implications for perceptual learning. Journal of Experimental Psychology: Animal Behavior Processes, 32, 145-155.

Honey, R. C., Bateson, P., \& Horn, G. (1994). The role of stimulus comparison in perceptual learning: An investigation with the domestic chick. Quarterly Journal of Experimental Psychology, 47B, 83-103.

Lombas, A. S., Alonso, G., \& Rodríguez, G. (2008). The influence of comparison between similar stimuli on the effectiveness of their unique features. Behavioural Processes, 78, 112-116.

McLaren, I. P. L., \& Mackintosh, N. J. (2000). An elemental model of associative learning: I. Latent inhibition and perceptual learning. Animal Learning \& Behavior, 28, 211-246.

Mondragón, E., \& Hall, G. (2002). Analysis of the perceptual learning effect in flavour aversion learning: Evidence for stimulus differentiation. Quarterly Journal of Experimental Psychology, 55B, 153-169.

Rodríguez, G., \& Alonso, G. (2004). Perceptual learning in flavoraversion learning: Alternating and blocked exposure to a compound of flavors and to an element of that compound. Learning \& Motivation, 35, 208-220.

Rodríguez, G., \& Alonso, G. (2008). Stimulus comparison in perceptual learning: Roles of sensory preconditioning and latent inhibition. Behavioural Processes, 77, 400-404.

Saldanha, E. L., \& Bitterman, M. E. (1951). Relational learning in the rat. American Journal of Psychology, 64, 37-53.

SPENCE, K. W. (1936). The nature of discrimination learning in animals. Psychological Review, 43, 427-449.

Sutherland, N. S., \& Mackintosh, N. J. (1971). Mechanisms of animal discrimination learning. New York: Academic Press.

Symonds, M., \& HALL, G. (1995). Perceptual learning in flavor aversion conditioning: Roles of stimulus comparison and latent inhibition of common stimulus elements. Learning \& Motivation, 26, 203-219.

Wills, S., \& MaCKINTOSH, N. J. (1999). Relational learning in pigeons? Quarterly Journal of Experimental Psychology, 52B, 31-52.

(Manuscript received June 6, 2007; revision accepted for publication September 26, 2007.) 